\title{
Liquefaction of Low-Rank Coals with Hydriodic Acid and Microwaves
}

\author{
J osé M. Andrés,* Ana C. Ferrando, and Pedro Ferrer \\ Instituto de Carboquímica, CSIC, Poeta Luciano Gracia 5, 50015-Zaragoza, Spain \\ Received September 9, 1997. Revised Manuscript Received J anuary 19, 1998
}

\begin{abstract}
A novel method for the chemical liquefaction of low-rank coals in relatively moderate conditions and short reaction times is presented. Using concentrated hydriodic acid as the reaction medium, microwaves as the energy source, and reaction times of $20 \mathrm{~min}$ at $230{ }^{\circ} \mathrm{C}$, up to $50 \%$ of the organic carbon of coal can be extracted with THF. The study of the process shows that temperature of the reaction and time are the main factors affecting the desulfurization process. The redox potential and the nucleophilicity of the concentrated hydriodic acid were the driving force of the reaction as experiments with other acids or lower acid concentrations gave worse results.
\end{abstract}

\section{Introduction}

Liquefaction of coal aims to obtain liquid, or solventsoluble, molecules by treating coal under various conditions. ${ }^{1}$ Given the polymeric nature of coal, bridges have to be broken in order to obtain fragments of an appropriate size to be solubilized by organic solvents. Moreover, hydrogenation is required when coal liquefaction extracts are intended for their use as transportation fuels. Most of the hitherto published work deals with hard coals, where $\mathrm{CH}_{2}$ links have to be broken, which requires strong reaction conditions, like high temperatures $\left(300-400{ }^{\circ} \mathrm{C}\right.$ ) and high hydrogen pressures (beyond 100 bar). The advantage of applying a treatment with hydriodic acid to low-rank coals lies in the fact that ester and ether bonds account for an important part of the structural links that keep coal constituents together. Hydroiodic acid can break these links under moderate reaction conditions to give smaller fragments, soluble in organic media, even though these fragments will contain high amounts of oxygen and will not be suitable for direct use as transportation fuels.

In a previous paper ${ }^{2}$ we showed that treating coal with hydriodic acid under microwaves in a sealed reactor under a reducing atmosphere removes sulfur from the coal. In this paper we analyze the effect of such a treatment on liquefaction yields and extract quality. We have found that the treatment is effective to solubilize up to the $50 \%$ of the organic carbon of lowrank coals, with reduction of the sulfur content of the extract.

\section{Experimental Section}

The experimental methodol ogy was described in a previous paper. ${ }^{2}$ Additionally, el emental oxygen analyses using a CarloErba 1180 elemental analyzer were obtained for the reaction

(1) Gorin, E. F undamentals of Coal Liquefaction. In Chemistry of Coal Utilization; Elliott, M. A., Ed.; J ohn Wiley \& Sons: New York, 1981; p 1845.

(2) Andrés, J . M.; Ferrando, A. C.; Membrado, L. Energy Fuels 1996, $10,425-30$. products. Sulfur present in the products as elemental sulfur was analyzed using the method developed for hydrocarbons. ${ }^{3}$ In summary, elemental sulfur is extracted from coal by sonication with cyclohexane, sodium cyanide is added to obtain sodium thiocyanate ion, and after addition of $\mathrm{Fe}^{2+}$ to obtain the red complex, it is analyzed colorimetrically. For subsequent calculations, elemental sulfur was accounted independently of the extract, as it can be easily removed from the product of reaction.

The extraction of the coals was carried out by sonication, using Gooch crucibles and a ultrasonic horn (Branson Sonifier 450 ). One gram of product was suspended in $40 \mathrm{~mL}$ of tetrahydrofuran in a crucible and sonicated for $5 \mathrm{~min}$ at low power level, enough to guarantee thorough mixing. After sonication, the mixture was filtered and new solvent was added to the crucible for further extraction. The solvent additionsonication-filtration cycle was repeated until no color was observed in the filtrate.

Concerning the extracts, it was not possible to obtain reliable analyses of them due to their sticky and heterogeneous nature once dried, and due to the presence of elemental sul fur. Therefore, the elemental composition of the extracts was calculated from mass balances. This method proved useful and the results obtained were in the range obtained for the extracts with a narrower confidence interval.

The quality of the extracts was studied using a modified saturated-aromatic-resins-asphaltene or polar (SARA) method. ${ }^{4}$ SARA-like analyses of the extracts were performed using a TLC-FID analyzer (I atroscan Mark V) using three consecutive development steps with (a) $5 \%$ dichloromethane in methanol for $3 \mathrm{~min}$, (b) toluene for $15 \mathrm{~min}$, and (c) hexane for $30 \mathrm{~min}$. Saturated compounds were found in low amounts and accounted together with aromatics. Elemental sulfur appears at $\mathrm{Rf}$ values close to that of aromatic compounds and will be accounted with saturates plus aromatics.

Characteristics of the coals used for this study are given in Table 1. Most of the research was carried out on the Sierra de Arcos (SA) coal, which contains noticeable amounts of carbonates and sulfates, so a soft demineralization with concentrated hydriodic acid at $25^{\circ} \mathrm{C}$ for $2 \mathrm{~h}$ was performed.

(3) Bartlett, J . K.; Skoog, D. A. Anal. Chem. 1954, 26, 1008-1011.

(4) Vela, J.; Cebolla, V. L.; Membrado, L.; Andrés, J. M. L. Chromatogr. Sci. 1995, 33, 417-425. 
Table 1. Composition of the Coals Used in This Work

\begin{tabular}{llll}
\hline & $\begin{array}{c}\text { Sierra de } \\
\text { Arcos }\end{array}$ & $\begin{array}{c}\text { Pozo } \\
\text { Pilar }\end{array}$ & $\begin{array}{c}\text { Virgen del } \\
\text { Pilar }\end{array}$ \\
\hline $\begin{array}{l}\text { proximate analysis (\%) } \\
\text { moisture }\end{array}$ & 6.4 & 4.8 & 6.1 \\
$\quad$ ash & 31.0 & 47.2 & 21.9 \\
elemental analysis (\%) & & & \\
$\quad$ carbon & 40.4 & 31.1 & 46.3 \\
$\quad$ hydrogen & 3.2 & 2.6 & 4.2 \\
$\quad$ nitrogen & 0.4 & 0.5 & 0.8 \\
$\quad$ sulfur & 6.3 & 5.3 & 9.3 \\
sulfur forms (\%) & & & \\
$\quad$ sulfate & 1.6 & 1.4 & 0.8 \\
$\quad$ pyritic & 1.9 & 2.6 & 0.3 \\
$\quad$ organic (diff) & 2.8 & 1.3 & 8.2 \\
extract (\%) & 2.5 & 4.2 & 10.8
\end{tabular}

Subsequent results and mass balances are referenced to this demineralized coal.

\section{Results and Discussion}

While hydriodic acid was the first reagent reported in coal liquefaction, 5 its use was discontinued. To the best of our knowledge, the only contributions to coal liquefaction with hydriodic acid are those of Van Krevel en ${ }^{6}$ using hydriodic acid at high temperatures and hydrogen pressures, out of the scope of the present work.

Hydroiodic acid has been used in organic chemistry mainly as a reagent for ether bond cleavage. Other interesting reactions of this reagent toward oxygenated functional groups can be found in organic chemistry textbooks. ${ }^{7,8}$ The expected effect of the reagent on the components of coal can be summarized as follows:

(a) Inorganic matter. Minerals commonly found in coal, like quartz or some clays, are insensitive to acid attack, carbonates and sulfates being the only ones easily removed by acid washing. In addition, we have shown that hydriodic acid is capable of removing pyritic sulfur from coal. ${ }^{2}$ The primary effect to be considered in coal liquefaction is the reduction of the amount of mineral matter left after reaction.

As most of the reactions were performed at temperatures above $200{ }^{\circ} \mathrm{C}$, decomposition of hydrogen iodide to iodine and hydrogen has to be taken into account. There is also an interesting reaction involving hydrogen sulfide and iodine:

$$
\mathrm{H}_{2} \mathrm{~S}+\mathrm{I}_{2} \leftrightharpoons 2 \mathrm{HI}+\mathrm{S}^{(0)}
$$

This reaction leads to formation of elemental sulfur in the reaction product, which will be extracted by organic solvents together with the extract and has to be accounted for.

(b) Organic matter. The most important reactions that can be considered taking place on coal are

$$
\begin{aligned}
& \text { cleavage of ethers: } \phi-\mathrm{O}-\mathrm{CH}_{2}-\mathrm{R}^{\prime}+\mathrm{HI} \rightarrow \\
& \qquad \phi-\mathrm{OH}+\mathrm{R}^{\prime}-\mathrm{CH}_{2}-\mathrm{I}
\end{aligned}
$$

(5) Berthelot, M. P. E. Bull. Soc. Chim. 1869, 11, 278.

(6) Van Krevelen, D. W. Coal, 3rd ed.; Elsevier: Amsterdam, 1993; pp 271-2.

(7) March, J. Advanced Organic Chemistry; International Student Edition; McGraw-Hill International Book Co.: Auckland, New Zealand, 1977.

(8) Allinger, N. L., et al. Organic Chemistry; Worth Publishers Inc., New York, 1971. cleavage of esters: $\mathrm{R}-\mathrm{COO}-\mathrm{R}^{\prime}+\mathrm{H}_{2} \mathrm{O}+$

$$
\mathrm{HI}_{\text {cat.am. }} \rightarrow \mathrm{R}-\mathrm{COOH}+\mathrm{R}^{\prime}-\mathrm{OH}
$$

halogenation of alcohols: $\mathrm{R}-\mathrm{OH}+\mathrm{HI} \rightarrow$

$$
\mathrm{R}-\mathrm{I}+\mathrm{H}_{2} \mathrm{O}
$$

reduction of alkyl iodides: $\mathrm{R}-\mathrm{I}+\mathrm{HI} \rightarrow \mathrm{R}-\mathrm{H}+\mathrm{I}_{2}$

Besides, $\mathrm{HI}$ can reduce other less common functional groups in coal or combinations of them, like glycols, benzyls, or aryl ketones. On the other hand, the reagent does not attack aromatics, phenols, and related substances.

Organic sulfur compounds do not behave like their oxygenated counterparts, with sulfides and mercaptans being resistant to cleavage by $\mathrm{HI}$, a facile reaction for the analogous oxygenated compounds like ethers and alcohols. The most important reaction of $\mathrm{HI}$ with sulfur compounds (in the context of this work) is

reduction of sulfoxides: $\mathrm{R}-\mathrm{SO}-\mathrm{R}^{\prime}+2 \mathrm{HI} \rightarrow$

$$
\mathrm{R}-\mathrm{S}-\mathrm{R}^{\prime}+\mathrm{H}_{2} \mathrm{O}+\mathrm{I}_{2}
$$

There is a caveat, however: most organic reactions involving $\mathrm{HI}$ are usually carried out using small amounts of reagent dissolved in an organic media, typically acetic acid, and at lower temperatures and pressures than those used in the present work.

When applied to low-rank coals, ether and ester cleavage can be expected, yielding phenols and/or aliphatic iodides, which, in some cases, can be dehalogenated. With regard to sulfur-containing functionalities, sulfides and mercaptans usually require strong reducing agents for their reduction so we cannot infer from literature data if concentrated hydriodic acid could break them.

Experimental work was planned using a factorial design to gain information about the reaction conditions to be used in subsequent experiments. From the obtained results, a base experiment with the following conditions was chosen: $1000 \mathrm{~W}$ microwave power for 5 min, temperature of $230^{\circ} \mathrm{C}$, atmosphere of $\mathrm{Ar}$ with 5 $\mathrm{mL}$ of $\mathrm{H}_{2}$ injected after reactor closure, and $75 \mathrm{~mL}$ of $\mathrm{HI}$ for the treatment of $5 \mathrm{~g}$ of coal in the $500 \mathrm{~mL}$ reactor. As discussed in a previous work, ${ }^{2}$ although the treatment with hydriodic acid causes material losses, those of carbon and heating value are small, so the amount of lost organic matter should be low. More important losses are found for the total amount of product recovered and the hydrogen content, which we attribute to a deeper attack on the mineral matter of the coal than that reached by the soft demineralization previously performed. Since the most important present mineral species are silicate minerals like kaolinite and montmorillonite, the attack will collapse their structure, with removal of cations and structural water, which would account for the concurrent decrease of the hydrogen content.

As there are several different reactions involved in the treatment, the influence of temperature in the process was the first variable to study, all other parameters being kept at their base value. Total and elemental mass balances and IR spectra of the reaction products together with estimated composition and SARA analyses of the extracts for experiments at various 

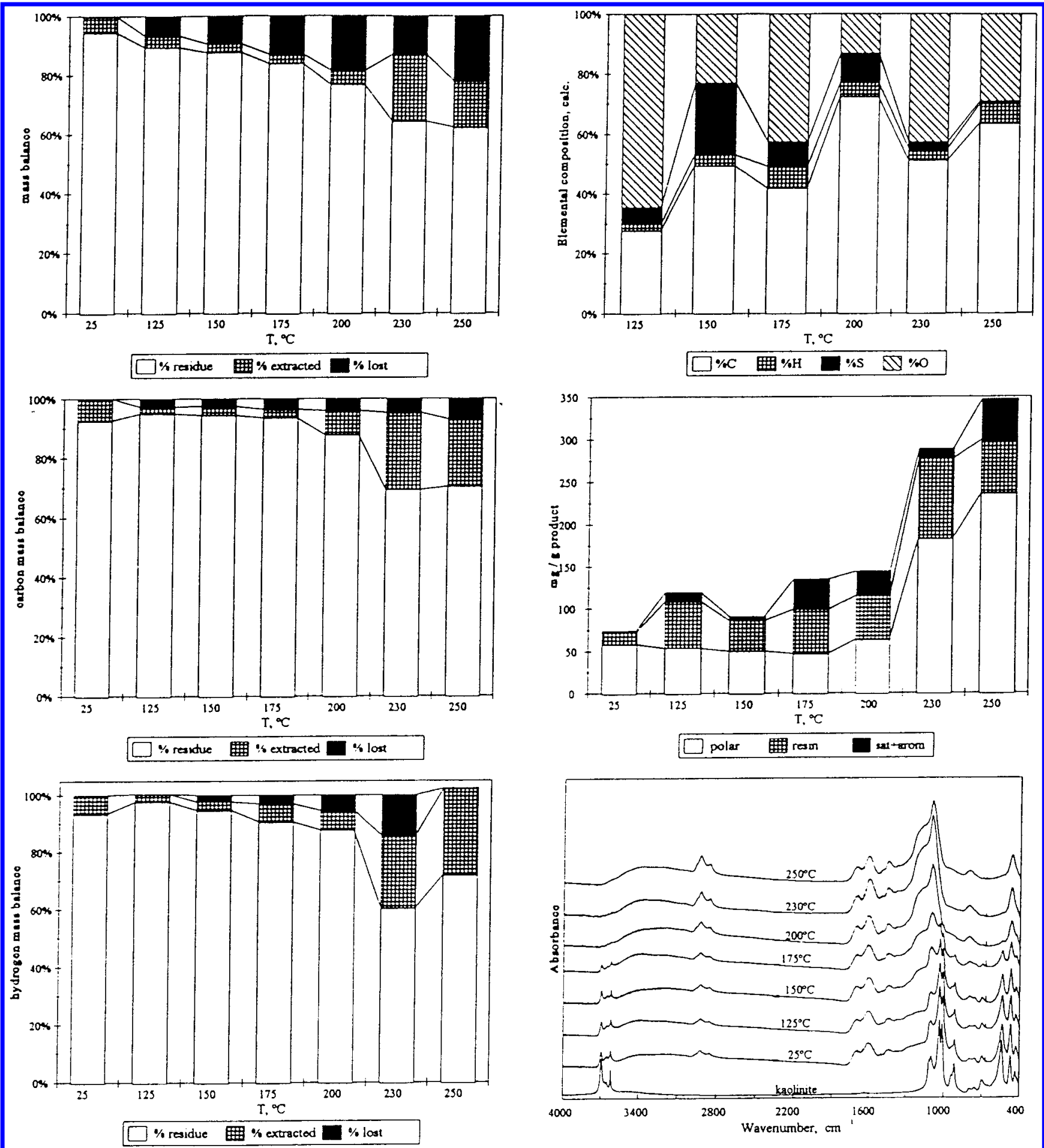

Figure 1. Influence of temperature in the treatment of coal with hydriodic acid. Mass, carbon and hydrogen balances for the reaction, infrared spectra of the obtained products and calculated el emental composition and SARA analyses of the extracts.

temperatures are shown in Figure 1. The total mass balance shows that an increase in the temperature of reaction increases the yield of extraction as well as the losses of matter, especially for temperatures higher than $200^{\circ} \mathrm{C}$. One point to note is that gas analyses showed no traces of organic gases, carbon dioxide and hydrogen sulfide being the only detected gaseous reaction products. Elemental mass balances showed that carbon losses increased slightly with reaction temperature, so we can infer that organic matter losses are small and can be due to decarboxilation or to the waste of watersoluble molecules as a result of the hydrolysis reaction.
Also, the behavior of carbon shows that temperatures higher than $200{ }^{\circ} \mathrm{C}$ are required to improve the yield of extraction. The most severe losses correspond to sulfur compounds, specifically pyritic sulfur. The behavior of hydrogen is interesting as an indication of the reactions taking place in the process. Increasing temperature from $125^{\circ} \mathrm{C}$ (near $\mathrm{HI}$ boiling point of $127^{\circ} \mathrm{C}$ ) to $230^{\circ} \mathrm{C}$ increases hydrogen losses as well as the amount of hydrogen transferred to the extract, but the trend is reversed when a temperature of $250^{\circ} \mathrm{C}$ is used, with a net increase of hydrogen content. The calculated composition of the extracts reflects the different reaction 

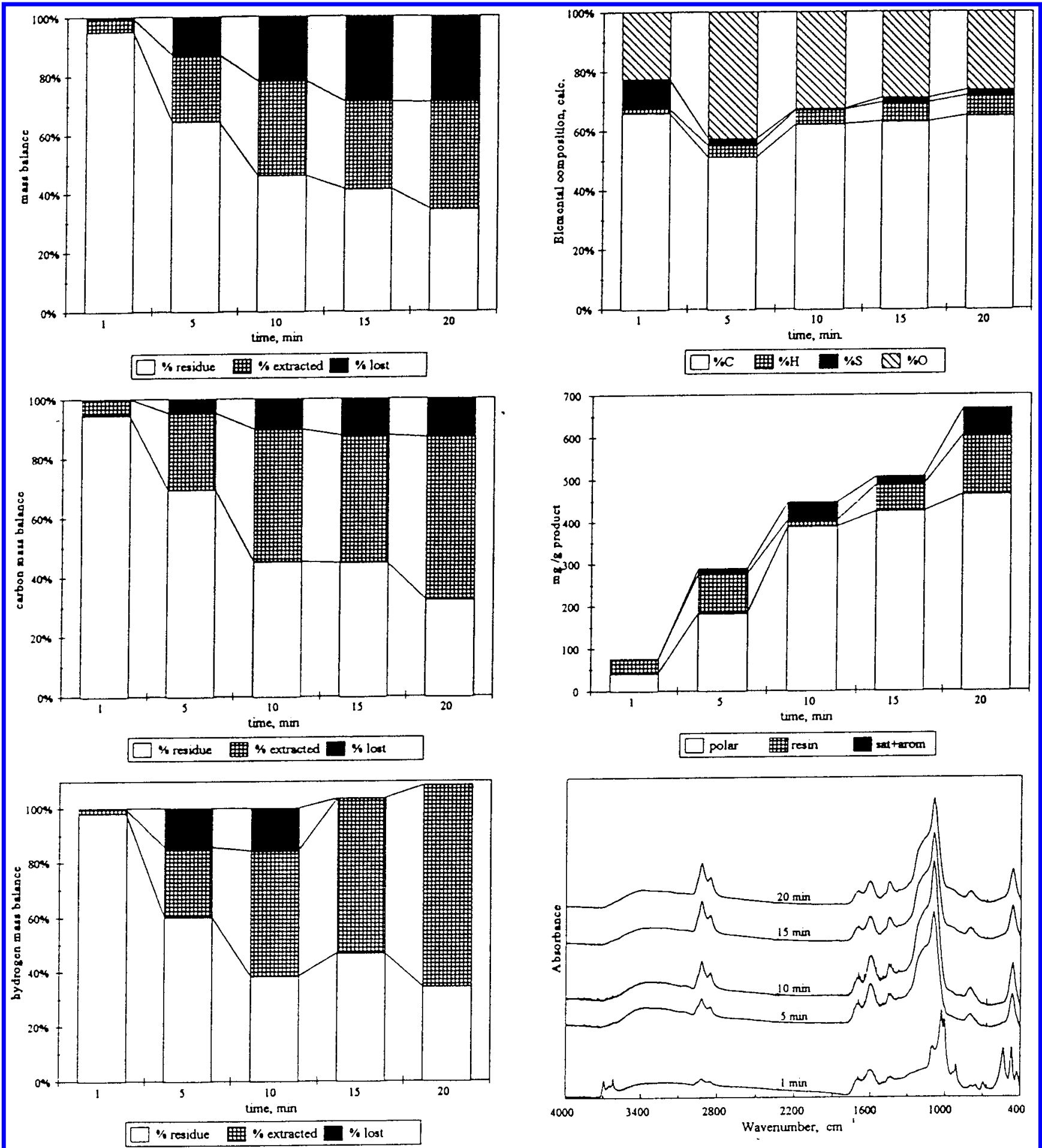

Figure 2. Effect of time in the hydriodic acid processing of coal. Mass, carbon, and hydrogen balances for the reaction, infrared spectra of the obtained products, and calculated elemental composition and SARA analyses of the extracts.

pathways that can take place in good agreement with SARA analysis.

Infrared spectra of the obtained products are shown in Figure 1, normalized and baseline corrected. Besides the destruction of the structure of kaolinite, as reflected in the disappearance of its characteristic absorptions, wide bands from 1150 to $1250 \mathrm{~cm}^{-1}$ and at $3350 \mathrm{~cm}^{-1}$ imply the formation of $\mathrm{COOH}$ and $\mathrm{OH}$ functional groups by hydrolysis of ester and ether bonds. Another interesting feature is the absorbance increase shown by the bands at $1440 \mathrm{~cm}^{-1}$ and from 2850 to $2960 \mathrm{~cm}^{-1}$, corresponding to more aliphatic moieties formed and extracted as the temperature of the reaction increases.

While microwaves cause a quick and effective heating of aqueous solutions, $5 \mathrm{~min}$ is too short a time for organic reactions, especially those dealing with solidliquid mixtures. A subsequent series of experiments was carried out to analyze the influence of time in the reaction yields, keeping the other reaction parameters at their base values (specifically, temperature was kept at $230{ }^{\circ} \mathrm{C}$ ) and varying time of reaction from 0 to 20 min in 5 min increments. The mass balances for these 

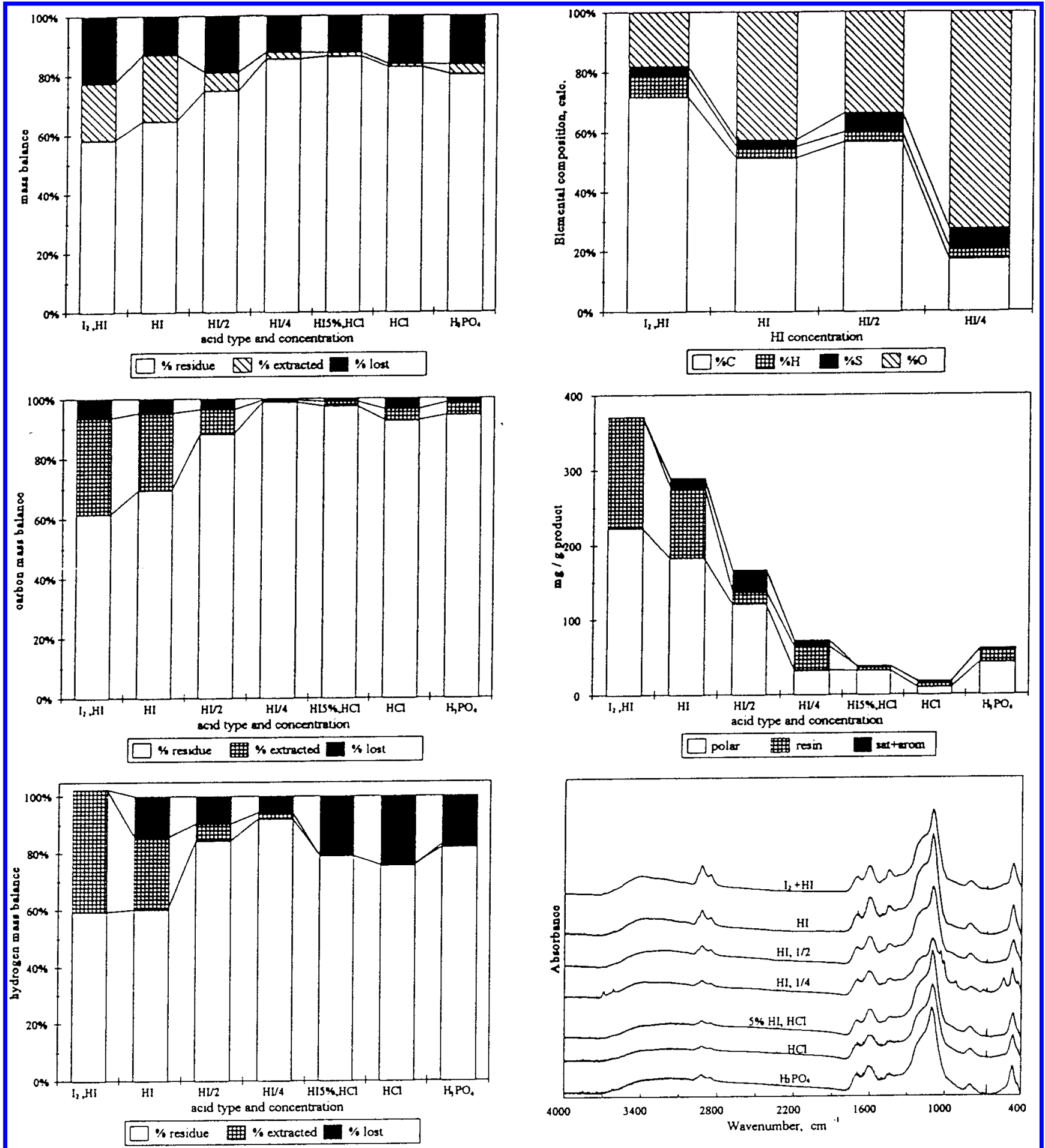

Figure 3. Effect of the kind of acid and concentration on the treatment. Mass, carbon and hydrogen balances for the reaction, infrared spectra of the obtained products, and calculated elemental composition and SARA analyses of the extracts.

experiments are shown in Figure 2, which demonstrates the pronounced effect of time on the reaction yield. Increasing reaction times increase extract yields as well as losses, as a consequence of the reactions taking place in greater extension. The main point seems to be that the extracts are also reactive to $\mathrm{HI}$, as there is a continuous increase in losses with reaction time. In this way, the yield of extract does not increase regularly with reaction time but, as observed for mass and $C$ balances, a change from 10 to 15 min causes a reduction of the amount of extract and increases losses. Sulfur is heavily removed at longer times but the percentage of sulfur in the extract seems to increase with reaction time. Hydrogen presents a behavior already shown when establishing the temperature effect, with a neat uptake for longer times of reaction. One point in case is that hydrogen seems to be incorporated to the residua besides the incorporation into the extract, as shown by the $\mathrm{H}$ mass balance. The calculated elemental composition of the extract shows that when increasing the reaction time carbon, hydrogen and sulfur content grow, with a lowering in the content of oxygen and iodine (by difference). This leads to higher quality extracts as reflected by the SARA analysis, with increasing amounts 


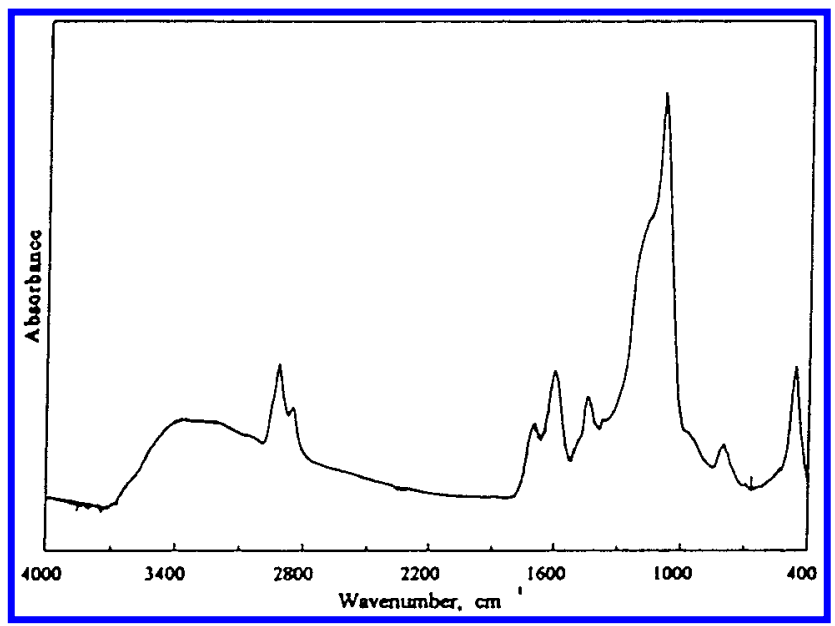

Figure 4. IR spectrum of a representative extract.

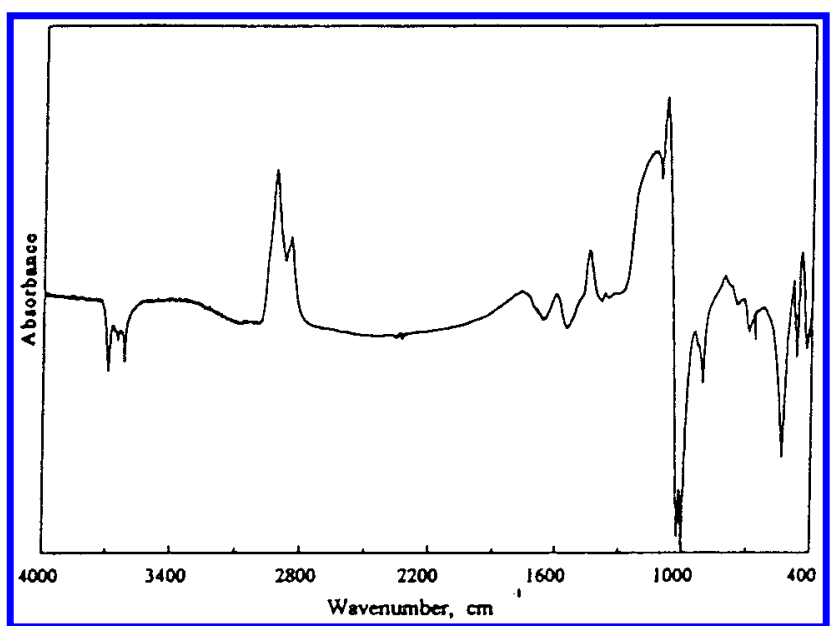

Figure 5. IR calculated cross-correlation spectra with extract yield.

of resins and saturated plus aromatics (the elemental $S$ content is very low for long reaction times) and less polar substances, indicative of extensive degradation to lighter fragments with lesser polarity. Close inspection of the IR spectra confirms the above-mentioned transformations under the influence of temperature, with emphasis on the absorbance increase for the bands at $1440 \mathrm{~cm}^{-1}$ and from 2850 to $2960 \mathrm{~cm}^{-1}$, indicative of an increment in aliphatic groups in the product.

The last set of experiments was devoted to determine if other acids besides hydriodic acid could be used and what effect the concentration of this acid has on the extraction yields. For comparison purposes, concentrated hydrochloric and orthophosporic acids were used, all other parameters being kept at their base level. As Figure 3 shows, neither hydrochloric nor orthophosphoric acid gave good results concerning liquefaction of coal. The same is true for diluted hydriodic acid, with both $1 / 2$ and $1 / 4$ dilutions yielding poor results, as well as the $5 \%$ hydriodic acid dilution with hydrochloric acid to maintain high acidity. It seems that the reaction pathways require concentrated hydriodic acid to progress. It is worth to mentioning that iodine plays an important role in the reaction as evidenced by an experiment carried out with $0.5 \mathrm{~g}$ of iodine added to concentrated hydriodic acid. While the net amount of extract is lower than that obtained for concentrated hydriodic acid, the amount of carbon transferred to the extract is higher
Table 2. Application of the HI Treatment to Different Coals

\begin{tabular}{lccccccc}
\hline \multicolumn{1}{c}{ coal } & yielda $^{\mathrm{C}}$ & $\begin{array}{c}\mathrm{C} \\
(\%)\end{array}$ & $\begin{array}{c}\mathrm{H} \\
(\%)\end{array}$ & $\begin{array}{c}\mathrm{O} \\
(\%)\end{array}$ & $\begin{array}{c}\mathrm{S}^{(0)} \\
(\%)\end{array}$ & $\begin{array}{c}\mathrm{S}_{\text {organic }} \\
(\%)\end{array}$ & $\begin{array}{c}\text { extracta }^{\mathrm{O}} \\
(\%)\end{array}$ \\
\hline Sierra de Arcos & 75.9 & 47.6 & 3.2 & 3.7 & 0.3 & 3.4 & 19.6 \\
Virgen del Pilar & 75.4 & 50.9 & 4.7 & 9.7 & 0.2 & 6.4 & 39.6 \\
Pozo Pilar & 67.7 & 43.1 & 3.6 & 9.3 & 0.1 & 1.3 & 14.1
\end{tabular}

a Referenced to raw coal.

using iodine as is the reaction extension (extraction yield plus losses), with a net uptake of hydrogen showing up in the extract. While iodine is able to remove sulfur to a great extent, the sulfur content of the extract is slightly higher than that obtained with hydriodic acid alone. The estimated composition of the extract indicates more carbon and less heteroatom content for the extract obtained with iodine but this is not reflected in the quality of the extract as indicated by SARA analysis, as similar amounts of resins are obtained in both cases. The importance of free iodine shows up in the IR spectra shown in Figure 4, where al iphatic bands at $1440 \mathrm{~cm}^{-1}$ and from 2850 to $2960 \mathrm{~cm}^{-1}$ grow with the addition of iodine to the reaction mixture.

The application of this method to the two other coals tested showed the real abilities of the method. The results, shown in Table 2 , reflect that the treatment with hydriodic acid allows an increase in the extraction yield for all the coals studied, this increase being more important for a less evolved, very special coal like Virgen del Pilar, from the Mequinenza basin.

There are two things common to all the reactions carried out: the IR spectra of the extraction residua are very similar to the IR spectra of the whole reaction products from which they are derived, possibly owing to incomplete reaction in the short times used, and the IR spectra of extracts show the same features as the IR spectrum shown in Figure 4, with some minor variation in the rel ative intensity of the bands. This IR spectrum shows the complexity and the high polarity of the extract.

The relevant changes produced by the treatment of coal with hydriodic acid in relation to the yield of extract are shown by the IR cross-correlation spectra2,9 in Figure 5. Three main points can be considered: first, the kaolinite structure is torn down as shown by the strong negative bands present in the calculated spectra. Second, there is an important increase in the absorbance of the bands in the $1100-1250 \mathrm{~cm}^{-1}$ region, related to $\mathrm{C}-\mathrm{O}$ bonds. Coupled with a small increase in the broad band at 3300 and $1600 \mathrm{~cm}^{-1}$ and with a shift in wavenumber from 1770 to $1720 \mathrm{~cm}^{-1}$, an increase in $\mathrm{OH}$ and $\mathrm{COOH}$ groups can be inferred, as could be expected in a hydrolysis process. Third, bands assigned to aliphatics at 1440 and $2850-2950 \mathrm{~cm}^{-1}$ show a very important increase in absorbance which seems to indicate that other reactions take place, reducing functional groups or eliminating heteroatoms from the product. Also, a selective extraction for the more smaller fragments can be inferred.

From the results shown above, the treatment with hydriodic acid is more than a simple hydrolysis process when temperatures of 230 or $250^{\circ} \mathrm{C}$ are used. In these

(9) Honigs, D. E.; Hieftje, G. M.; Hirschfeld, T. Appl. Spectrosc. 1984, 38, 317-322. 
conditions, a reduction or hydrogenation reaction takes place, causing the formation of new $\mathrm{C}-\mathrm{H}$ bonds and a neat hydrogen uptake. Possible reagents for this reduction are the hydriodic acid or hydrogen either introduced initially or arising from the thermal decomposition of hydriodic acid. This decomposition would also produce free iodine which can act as a hydrogenation catalyst ${ }^{5,10,11}$ even in the conditions used in this work, as evidenced by experiments with added iodine. In addition, carbon-sulfur bonds are broken by this reagent and carboxyl groups are removed as can be inferred from the results, but a more detailed study is required to

(10) Fraenkel, D., et al. Fuel 1991, 70, 64-73.

(11) J oseph, J . T.; Duffield, J. E.; Davidson, M. G. Energy Fueds 1992 6, 764-770. know which kind of $\mathrm{C}-\mathrm{S}$ bonds or $\mathrm{COOH}$ groups is more reactive toward hydriodic acid. On the other hand, from a coal liquefaction perspective, an extensive optimization of the process is needed to attain yields of extraction higher than those shown in the present work. For that matter, working at higher hydrogen pressures and longer times should all ow greater carbon recoveries and better extract characteristics, as those obtained here are too polar for its intended use.

Acknowledgment. This work was funded by the European Coal and Steel Community (ref. 9220-EC/759) and Spanish CICYT (ref. AMB92-1221-CE).

EF 9701807 